\title{
Gamma Oscillations and Stimulus Selection
}

\section{Christoph Börgers}

christoph.borgers@tufts.edu

Department of Mathematics, Tufts University, Medford, MA 02155, U.S.A.

\author{
Nancy J. Kopell \\ $n k @ . b u . e d u$ \\ Department of Mathematics and Center for Biodynamics, Boston University, \\ Boston, MA 02215, U.S.A.
}

More coherent excitatory stimuli are known to have a competitive advantage over less coherent ones. We show here that this advantage is amplified greatly when the target includes inhibitory interneurons acting via $\mathrm{GABA}_{\mathrm{A}}$-receptor-mediated synapses and the coherent input oscillates at gamma frequency. We hypothesize that therein lies, at least in part, the functional significance of the experimentally observed link between attentional biasing of stimulus competition and gamma frequency rhythmicity.

\section{Introduction}

Biasing of competition in favor of an attended stimulus is known to be correlated with enhanced gamma band $(30-80 \mathrm{~Hz})$ synchronization (Fries, Schröder, Roelfsema, Singer, \& Engel, 2002; Gruber, Müller, Keil, \& Elbert, 1999; Taylor, Mandon, Freiwald, \& Kreiter, 2005). We propose here a possible link between gamma rhythmicity and selectivity: a coherent input oscillating at gamma frequency can be highly effective at preventing less coherent competing inputs from being noticed when the target network includes inhibitory interneurons acting via $\mathrm{GABA}_{\mathrm{A}}$-receptor mediated synapses. Two factors contribute to this effect. First, inhibition in effect raises the leakiness of the target neurons, thereby greatly amplifying the known (Murthy \& Fetz, 1994; Singer, 1999) advantage of a more coherent excitatory input $A$ over a less coherent competitor $B$. A highly coherent stimulus can break through the inhibition generated in the target network, while less coherent competitors cannot. Second, when a gamma frequency train $A$ of excitatory input pulses entrains a target network of excitatory and inhibitory model neurons, the timing of the inhibitory spike volleys in the target favors $A$ over any competing pulse train $B$ that has a different frequency or phase.

Our work on this subject was inspired by a recent paper by Fries (2005), who suggested that phase locking with the target should lend a competitive 
advantage to a stimulus. This idea is related to our second point above; we will show it to be valid (at least in our model) in the presence of inhibition in the target but not in its absence.

In section 2, we describe our models. In section 3.1, we present results of numerical simulations demonstrating the large competitive advantage of more coherent excitatory inputs to networks of excitatory and inhibitory model neurons over less coherent ones. In section 3.2, we analyze how synaptic inhibition within the target favors coherent inputs by effectively raising the leakiness of the target neurons-our first point above. In section 3.3, we discuss how the timing of inhibitory population spikes within the target neuron favors an entraining input over others-our second point. In section 3.4, we explain why suppression of a less coherent input train $B$ by a more coherent input train $A$ requires that $A$ oscillate at least at gamma frequency. We conclude section 3 with a brief discussion in section 3.5 of the robustness of our results with respect to parameter changes, heterogeneity in neuronal and network properties, and noise. Although incoherent input is less effective at eliciting responses, it can be effective at raising the excitability of target cells. In particular, an incoherent distractor may enable the inhibitory cells in the target network to fire. It is therefore possible for the incoherent distractor itself to enable the mechanism that prevents it from entraining the target network. This is illustrated by a computational example in section 4 . We summarize and discuss our results in section 5.

\section{Models}

The mechanisms studied here require no currents other than those in the classical Hodgkin-Huxley model (Hodgkin \& Huxley, 1952). In general, cortical neurons are capable of producing many other ionic currents. Somefor instance, hyperpolarization-activated inward currents such as $I_{h}$ (Lüthi \& McCormick, 1998)—are probably negligible during the sort of driven gamma activity considered here, since membrane potentials do not become sufficiently low. Others, for instance depolarization-activated slow outward currents such as $I_{M}$, may well be present and are in fact thought to be modulated by attention (Hasselmo \& McGaughy, 2003; Sarter, Givens, \& Bruno, 2001). However, we do not expect the presence of such currents to alter our main points fundamentally as long as the target neurons fire in response to input pulses, but not-or much less frequently-otherwise. Those aspects of the physiology that are important to our study should be well described by standard Hodgkin-Huxley equations, which in turn can often be well approximated by reduced equations such as integrate-and-fire models (Abbott, 1999; Latham, Richmond, Nelson, \& Nirenberg, 2000) or the theta model (Ermentrout \& Kopell, 1986; Gutkin \& Ermentrout, 1998; Hoppensteadt \& Izhikevich, 1997). In this letter, we primarily use theta neurons, since they have more realistic input response characteristics than integrate-and-fire neurons. However, for simplicity and transparency, some 
of our analysis will also be presented for linear integrate-and-fire neurons. For both theta and integrate-and-fire neurons, we model synapses in the standard conductance-based way.

2.1 Theta Neurons. In the theta model (Ermentrout \& Kopell, 1986; Gutkin \& Ermentrout, 1998; Hoppensteadt \& Izhikevich, 1997), a neuron is represented by a point $P=(\cos \theta, \sin \theta)$ moving on the unit circle. This is analogous to the Hodgkin-Huxley model, which represents a periodically spiking space-clamped neuron by a point moving on a limit cycle in a four-dimensional phase space. In the absence of synaptic coupling, the differential equation describing the motion of the point $P$ is

$$
\frac{d \theta}{d t}=1-\cos \theta+I(1+\cos \theta)
$$

Here $t$ should be thought of as time measured in milliseconds (Börgers \& Kopell, 2005, sec. 2.1) and $I$ as the analog of an external input current.

For a negative constant $I$, equation 2.1 has the two fixed points:

$$
\theta_{0}^{ \pm}= \pm 2 \arccos (1 / \sqrt{1-I})
$$

The fixed point $\theta_{0}^{-} \in(-\pi, 0)$ is stable, and $\theta_{0}^{+} \in(0, \pi)$ is unstable. As $I$ increases, the fixed points approach each other. As $I$ crosses 0 from below, a saddle-node bifurcation occurs: the fixed points collide at $\theta_{0}^{-}=\theta_{0}^{+}=0$, and there are no fixed points for $I>0$. For a theta neuron, to "spike" means, by definition, to reach $\theta=\pi$ (modulo $2 \pi$ ). The transition from $I<0$ to $I>0$ is the analog of the transition from excitability to spiking in a neuron. In this letter, we study effects of input coherence and therefore allow $I$ to be a function of time (see section 2.3).

The theta neuron is equivalent, up to a change of variable, to a quadratic integrate-and-fire neuron with threshold potential $V_{T}=+\infty$ and reset potential $V_{\text {reset }}=-\infty$. (For a more detailed discussion of this connection, see section 2.1 of Börgers \& Kopell, 2005.)

We turn now to a description of how we model synapses among theta neurons. To derive the terms in the differential equations that model synaptic interactions, we use the connection between the theta neuron and the quadratic integrate-and-fire neuron mentioned in the previous paragraph. For the quadratic integrate-and-fire neuron, synapses are modeled in the standard way: by adding terms of the form

$$
g_{s} s(t)\left(V_{r e v}-V\right)
$$

to the right-hand side of the equation governing $V$. The gating variable $s(t)$ rises rapidly when the presynaptic neuron spikes and decays exponentially 
thereafter; our specific choice of $s(t)$ will be stated at the end of this section. Using the change of variable that leads from the quadratic integrate-andfire neuron to the theta neuron, one obtains a model of synapses among theta neurons (for details, we refer again to Börgers \& Kopell, 2005). The equation of a theta neuron subject to an excitatory synaptic input becomes

$$
\frac{d \theta}{d t}=1-\cos \theta+\left(I+12 g_{s} s(t)\right)(1+\cos \theta)-g_{s} s(t) \sin \theta
$$

where $s(t)$ is the gating variable associated with the synapse. Similarly, the equation of a theta neuron subject to an inhibitory synaptic input is

$$
\frac{d \theta}{d t}=1-\cos \theta+\left(I-\frac{3}{2} g_{s} s(t)\right)(1+\cos \theta)-g_{s} s(t) \sin \theta
$$

The constants 12 and 3/2 in equations 2.4 and 2.5 depend on the synaptic reversal potentials $V_{\text {rev }}$ (see equation 2.3) assumed in the derivation, but their precise values do not appear to affect the results of this study in a qualitative way. When a theta neuron is subject to multiple synaptic inputs at the same time, the resulting terms are summed:

$$
\begin{aligned}
\frac{d \theta}{d t}= & -\cos \theta+\left(I+12 \sum_{i} g_{s, E, i} s_{E, i}(t)-\frac{3}{2} \sum_{j} g_{s, I, j} s_{I, j}(t)\right)(1+\cos \theta) \\
& -\left(\sum_{i} g_{s, E, i} s_{E, i}(t)+\sum_{j} g_{s, I, j} s_{I, j}(t)\right) \sin \theta .
\end{aligned}
$$

Here the subscripts $E$ and $I$ indicate conductances and gating variables associated with E-cells (labeled by $i$ ) and I-cells (labeled by $j$ ), respectively. In our computational simulations, $s(t)$ is a smooth function governed by

$$
\frac{d s}{d t}=-\frac{s}{\tau_{D}}+e^{-\eta(1+\cos \theta)} \frac{1-s}{\tau_{R}}
$$

where $\theta$ is associated with the presynaptic neuron. We always use $\eta=$ 5 and $\tau_{R}=0.1$. Thus, $s$ rises rapidly (but not instantaneously) toward 1 when $\theta \approx \pi$ modulo $2 \pi$ and decays exponentially with time constant $\tau_{D}$ otherwise. For E-cells, $\tau_{D}=2$, and for I-cells, $\tau_{D}=10$. Since we think of $t$ as time measured in milliseconds, these are approximately the decay time constants associated with AMPA and $\mathrm{GABA}_{A}$ receptor-mediated synapses, respectively. For analytic purposes (in particular in appendices $C$ and D), we simplify by assuming $s(t)$ to jump to 1 instantaneously when the presynaptic neuron spikes and to decay exponentially thereafter. 
2.2 Networks. We denote by $N_{E}$ and $N_{I}$ the number of E- and I-cells in the model network, respectively, and by $I_{E}$ and $I_{I}$ the drives to the Eand I-cells. $I_{E}$ and $I_{I}$ may be different for different neurons and will be time varying (see section 2.3). Connectivity is all-to-all, except in the simulation underlying Figure 10 (see section 3.5.5 and appendix A for details). We denote by $g_{I E}$ the sum of all conductances associated with inhibitory synapses acting on a given E-cell; thus, assuming all-to-all connectivity, an individual $\mathrm{I} \rightarrow$ E synapse has strength $g_{I E} / N_{I}$. Parameters $g_{I I}, g_{E I}$, and $g_{E E}$ are defined similarly. Throughout this letter, we assume that $g_{I E}=g_{I I}$ (but see the comment at the beginning of section 3.5.1); we denote the common value of these two parameters by $g_{I}$.

2.3 External Drives. We use oscillatory external drives of the form

$$
I(t)=C+Q\left[\sum_{k=-\infty}^{\infty} \frac{T}{\sqrt{2 \pi \sigma^{2}}} \exp \left(-\frac{(t-(\varphi+k) T)^{2}}{2 \sigma^{2}}\right)-1\right]
$$

Here $C$ is the constant component (the temporal average) of $I$. The term in brackets, multiplied by the constant $Q>0$, is the oscillatory component. The oscillation period is $T$, the width of the input pulses is $\sigma$, and $\varphi$ is a phase shift. The frequency of the input is

$$
f=\frac{1000}{T}
$$

The factor of 1000 is needed because we think of $T$ as measured in ms but want to think of $f$ as measured in $\mathrm{Hz}$, not $\mathrm{kHz}$. The temporal average of the oscillatory component (the term in brackets on the right-hand side of equation 2.6) can easily be shown to be zero. The oscillation amplitude depends on $T / \sigma$ and rapidly decreases with $T / \sigma$; for small $T / \sigma$, one must therefore choose a large value of $Q$ if one wants a sizable oscillation. Figure 1 shows two examples, one with $\sigma=2$ (sharp pulses, or high coherence) and the other with $\sigma=7$ (broad pulses, or low coherence).

Each neuron receives two input streams of the form 2.6-one referred to as the primary stimulus, or input $A$, and the other as the distractor, or input $B$. We use subscripts to indicate the input stream, the type of the neuron receiving the input (E or I), and the index of the neuron. For instance, the primary stimulus (stimulus $A$ ) to the $i$ th E-cell is denoted by

$$
\begin{aligned}
& I_{A, E, i}(t)=C_{A, E, i} \\
& \quad+Q_{A, E, i}\left[\sum_{k=-\infty}^{\infty} \frac{T_{A, E, i}}{\sqrt{2 \pi \sigma_{A, E, i}^{2}}} \exp \left(-\frac{\left(t-\left(\varphi_{A, E, i}+k\right) T_{A, E, i}\right)^{2}}{2 \sigma_{A, E, i}^{2}}\right)-1\right]
\end{aligned}
$$




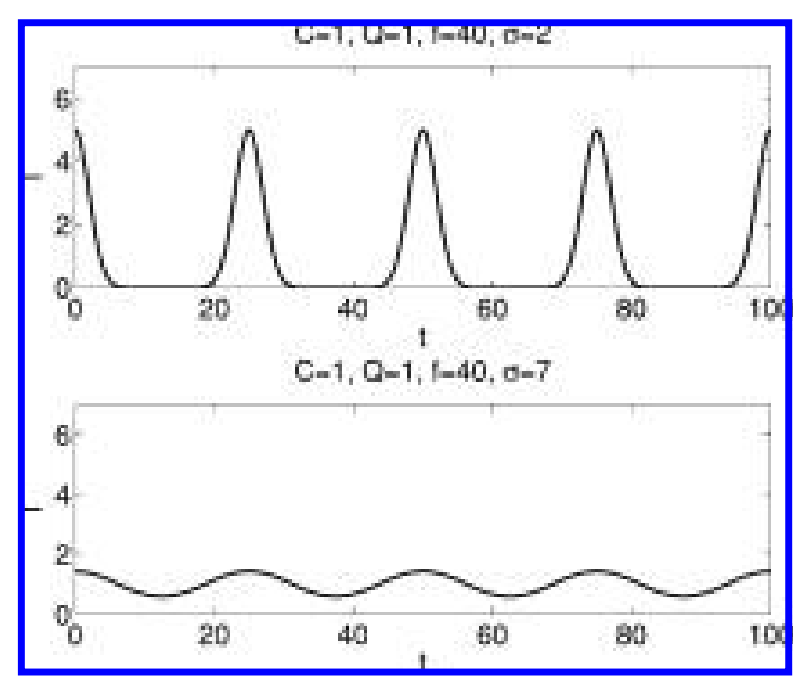

Figure 1: Examples of oscillatory inputs of the form 2.6.

and has frequency

$$
f_{A, E, i}=\frac{1000}{T_{A, E, i}} .
$$

2.4 Linear Integrate-and-Fire Neurons. For analysis purposes, we will also use linear integrate-and-fire neurons in this letter. Nondimensionalizing the membrane potential $V$ by appropriately shifting and scaling, we may assume in the linear integrate-and-fire model that the resting potential, in the absence of external drive, is $V_{R}=0$ and the spiking threshold is $V_{T}=1$. The time evolution of the membrane potential $V$ below threshold is then governed by an equation of the form

$$
\frac{d V}{d t}=-g_{m} V+I
$$

In this equation, $V$ is nondimensional, but $t$ is time, $g_{m}$ is the reciprocal of the membrane time constant (or equivalently, membrane conductance divided by capacitance), and $I$ is external input current times a (dimensional) constant.

We model synaptic input to linear integrate-and-fire neurons in the standard way:

$$
\frac{d V}{d t}=-g_{m} V+I+g_{s} s(t)\left(V_{r e v}-V\right)
$$




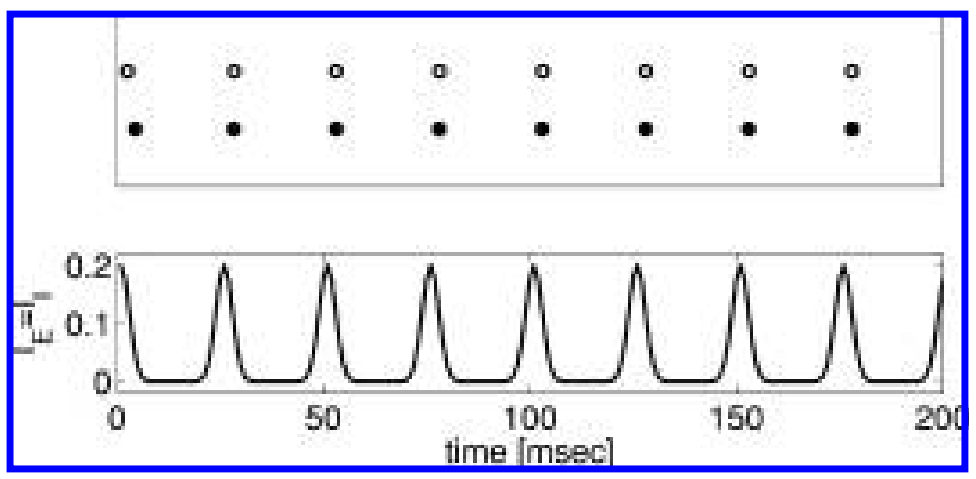

Figure 2: Periodic input pulses to both the E-cell and the I-cell entraining both cells. Spikes of the E-cell are indicated by open circles and spikes of the I-cell by filled circles.

Here $V_{\text {rev }}$ is the reversal potential of the synapse, $g_{s}>0$ is the maximum synaptic conductance divided by capacitance, and $s(t)$ is the gating variable associated with the synapse.

\section{Stimuli Oscillating Coherently at Gamma Frequency Render Incoherent Competitors Ineffective}

3.1 Simulations. We consider a target network consisting of a single E-cell and a single I-cell, with strong $\mathrm{E} \rightarrow \mathrm{I}, \mathrm{I} \rightarrow \mathrm{E}$, and $\mathrm{I} \rightarrow \mathrm{I}$ synapses but no $\mathrm{E} \rightarrow \mathrm{E}$ synapses. Figure 2 shows a simulation in which there is a $40 \mathrm{~Hz}$ sequence of tight input pulses $A$, acting on both target cells equally, without any distractor $B$. (The precise parameter values used in this and other simulations of this letter are listed in appendix A.) The target cells are entrained by $A$; their spikes occur shortly after the input pulse arrivals.

We now add a distracting stimulus $B$, somewhat stronger than $A$ on average but less coherent, oscillating at $25 \mathrm{~Hz}$. Figure $3 \mathrm{~A}$ shows the result. The distractor does not prevent the target network from following $A$ at $40 \mathrm{~Hz}$; it merely makes the rhythm of the target network slightly less regular. In Figure $3 \mathrm{~A}$, the distractor $B$ oscillates more slowly than the primary stimulus $A$, and the target network has only two cells. However, very similar results are obtained for distractors oscillating faster than $A$ (see Figure 3B for an example and section 3.5.4 for further numerical experiments concerning the distractor frequency).

One might first think that input $A$ dominates in Figure $3 \mathrm{~A}$ not because it is more coherent than input $B$ but simply because its amplitude is greater. Figure $3 \mathrm{C}$ demonstrates that this is not the correct interpretation. The figure shows a case in which the distractor $B$ is much stronger than $A$ on the temporal average and even slightly stronger in amplitude. The target is 


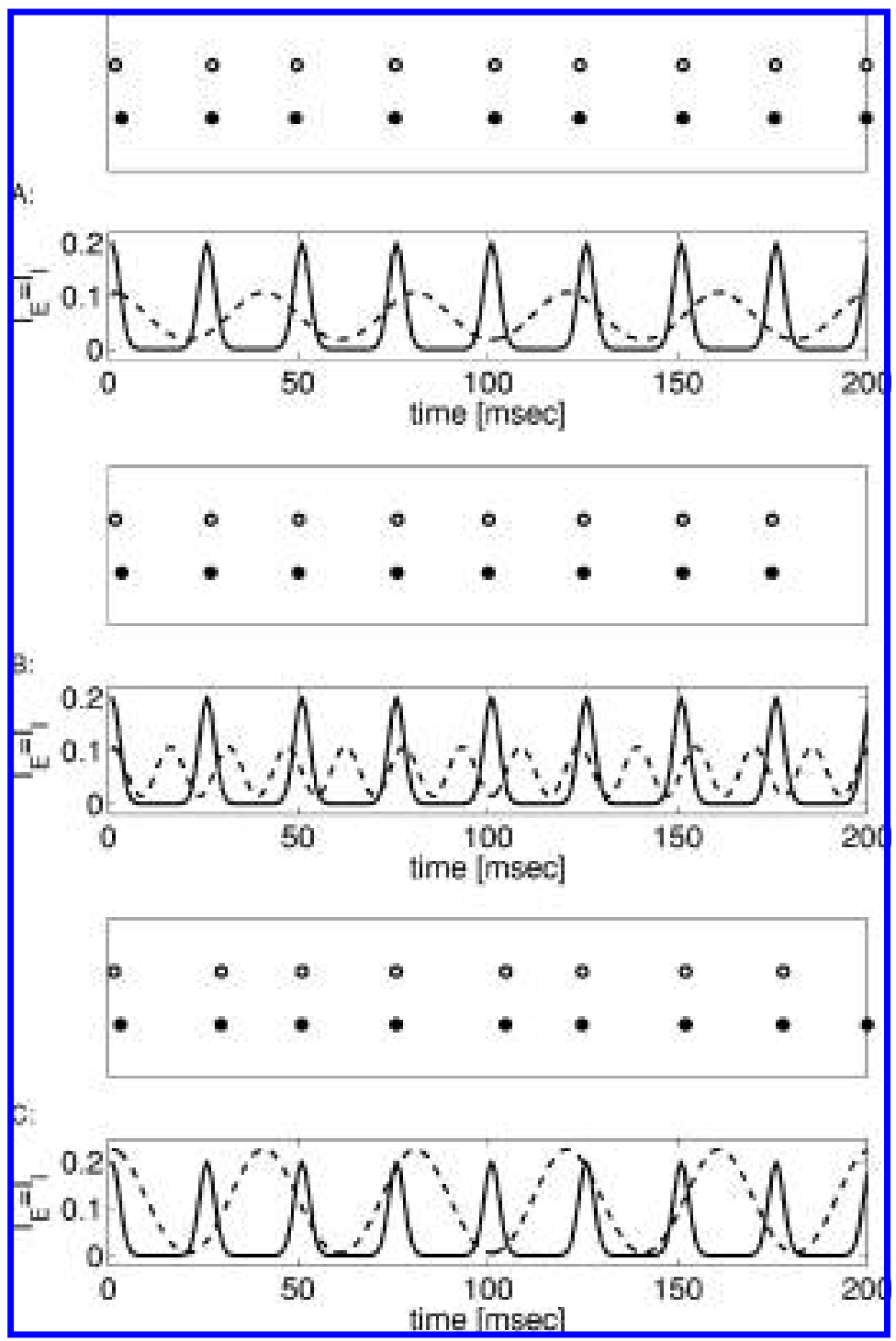

Figure 3: Two-cell target networks, consisting of an E-cell and an I-cell (spike times indicated by open and filled circles, respectively), driven by a coherently oscillating primary stimulus (solid) competing with a less coherently oscillating distractor (dashed). The distractor often has little effect even though its temporal average is greater than that of the primary stimulus (panel $A$ ). The distractor frequency may be lower than that of the primary stimulus $(A)$ or higher $(B)$. With strong local inhibition, this effect can even be seen when the distractor is so strong that its amplitude exceeds that of the primary stimulus $(C)$. 
still entrained by $A$. The precise parameter values used in the simulation are listed, as always, in appendix A. The inhibitory synapses are also much stronger here than in the previous simulation; if they were not strengthened, then the distractor would indeed be powerful enough in this example to prevent the entrainment of the target to input $A$.

We note that in the experiments of Figure 3 (and in all other numerical experiments of this letter), entrainment of the target by a stimulus means that the target cells promptly respond with a spike to each pulse of the stimulus, but do not spike otherwise.

\subsection{Inhibition Amplifies the Advantage of Coherent Input by Raising} the Effective Leakiness of the Target Neurons. Coherent (i.e., nearly simultaneous) excitatory input pulses targeting a leaky neuron are more likely to trigger a spike response than incoherent ones (Murthy \& Fetz, 1994; Singer, 1999). The advantage of coherent input can be amplified by synaptic inhibition, which can significantly raise the leakiness of the target neuron (Häusser \& Clark, 1997; Funabiki, Koyano, \& Ohmori, 1998; Pouille \& Scanziani, 2001; Grande, Kinney, Miracle, \& Spain, 2004). Here we present numerical and analytic results for theta and integrate-and-fire neurons confirming that this is an important effect.

3.2.1 Numerical Results for Theta Neurons. Figure 4A demonstrates that the well-known advantage of more coherent excitatory input pulses over less coherent ones alone falls far short of explaining the results of section 3.1. The figure shows the result of repeating the experiment of Figure 3A without the I-cell: the distractor now has a strong effect. Of course, if the distractor is reduced in strength far enough, it is ignored by the target even in the absence of inhibition. For instance, keeping $C_{B}$ and $Q_{B}$ equal to each other, one must reduce the common value of those two parameters to about 0.008 for the distractor to remain ineffective (see Figure 4B).

The timing of inhibition can play a role in suppressing the response to the distractor $B$ (see section 3.3), but the presence of inhibition alone often suffices to give the more coherent $A$ a decisive advantage over the less coherent $B$. For example, in the simulation of Figure $3 \mathrm{~A}$, the synaptic gating variable $s_{I}(t)$ associated with the I-cell oscillate, but if one replaces, in the code, $s_{I}(t)$ by its temporal average, the resulting figure is nearly indistinguishable from Figure 3A.

3.2.2 Analysis for Linear Integrate-and-Fire Neurons. We next present an analysis of the role of synaptic inhibition in lending an advantage to the more coherent input. To make our arguments as simple and transparent as possible, we begin with analysis for linear integrate-and-fire neurons, normalized as in equation 2.7. However, in section 3.2.3 and appendix B, we also outline the analysis for theta neurons. 


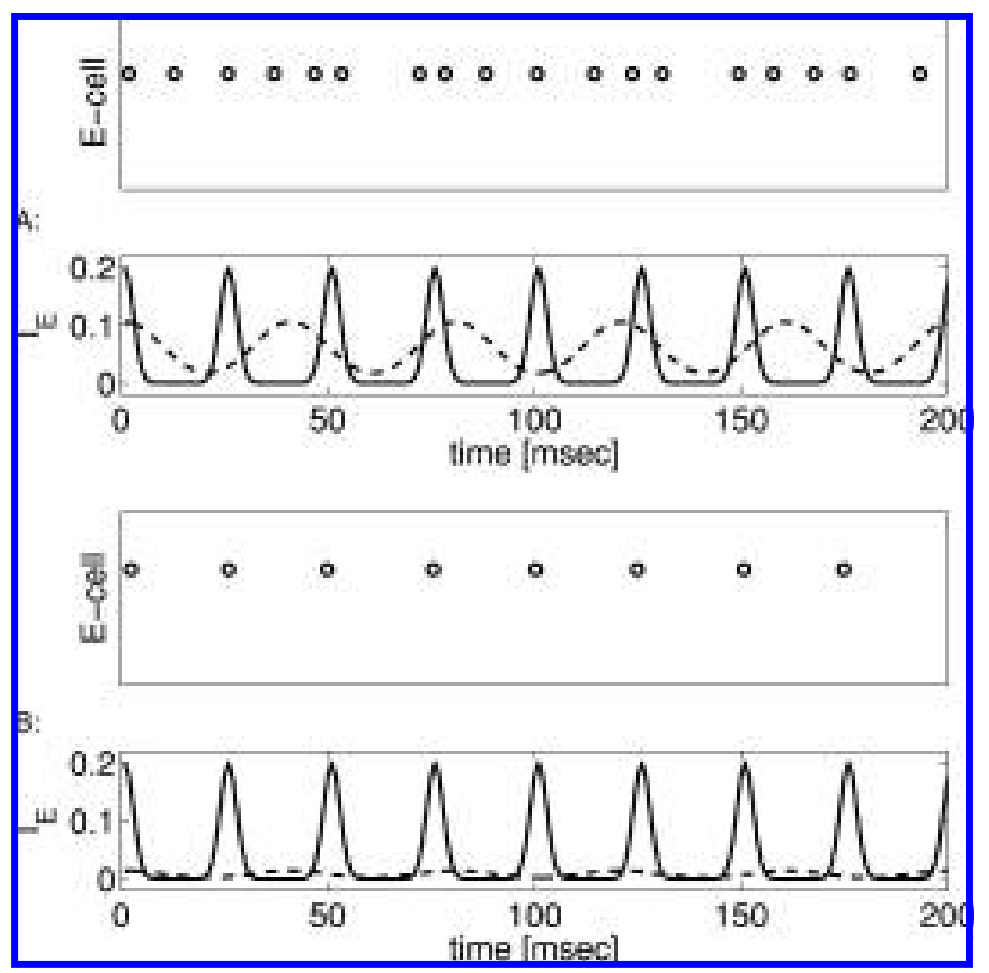

Figure 4: (A) The fact that coherent stimuli are more powerful than incoherent ones does not, by itself, explain Figure 3A. When inhibition is removed, the distractor has a strong effect. (B) For a weakly coherent distractor to remain ineffective in the absence of local inhibition, it must have very low strength.

Our starting point is a simple calculation demonstrating the intuitive and well-known fact that coherence makes excitatory input to an isolated target neuron more effective. The purpose of presenting this calculation here is to set the stage for a similar calculation with synaptic inhibition added.

Suppose that $V(0)=0$ and that during a brief time interval $\left[0, \tau_{J}\right]$, an input current $J>0$ is added to the right-hand side of equation 2.7:

$$
\frac{d V}{d t}=-g_{m} V+J \text { for } 0 \leq t \leq \tau_{J}
$$

We denote by

$$
q=\tau_{J} J
$$


the total amount of charge injected and ask whether the input pulse suffices to elicit a spike. Of course, the answer depends on $\tau_{J}$ and $J$, or equivalently, using equation 3.2 , on $\tau_{J}$ and $q$. The solution of equation 3.1 with $V(0)=0$ is

$$
V(t)=\frac{J}{g_{m}}\left(1-e^{-g_{m} t}\right)=\frac{q}{g_{m} \tau_{J}}\left(1-e^{-g_{m} t}\right),
$$

so a spike is elicited if and only if

$$
\frac{q}{g_{m} \tau_{J}}\left(1-e^{-g_{m} \tau_{J}}\right) \geq 1
$$

or

$$
q \geq \frac{g_{m} \tau_{J}}{1-e^{-g_{m} \tau_{J}}}
$$

To interpret this inequality, we note that the membrane time constant of our model neuron is $\tau_{m}=1 / g_{m}$. (See the discussion of physical dimensions following equation 2.7). Therefore, the quantity $g_{m} \tau_{J}$ appearing in equation 3.3 equals $\tau_{J} / \tau_{m}$, the pulse duration measured in membrane time constants. Since $x /\left(1-e^{-x}\right)$ is an increasing function of $x>0$, equation 3.3 shows that the total charge needed to elicit a spike is an increasing function of the pulse duration, measured in membrane time constants. The advantage of briefer, higher-amplitude input pulses over broader, lower-amplitude ones is amplified when the neuron is made leakier, that is, when $\tau_{m}$ is lowered.

Inequality 3.3 defines the region $\mathcal{S}_{g_{m}}$ of pairs $\left(q, \tau_{J}\right)$ for which a spike is elicited. For $g_{m}=0.2, \mathcal{S}_{g_{m}}$ is depicted in Figure 5A; it is the union of the light and dark shaded regions in the figure. (The significance of the two grades of shading will be explained shortly.) The fact that the boundary of the shaded region (indicated in bold in Figure 5) is slanted to the right means that more charge $q$ is needed to elicit a spike when $\tau_{J}$ is greater; in other words, excitatory input is less effective when delivered in a broader pulse.

The advantage of more coherent input pulses over less coherent ones is raised by synaptic inhibition. To understand this, we now consider an integrate-and-fire neuron subject to constant synaptic inhibition:

$$
\frac{d V}{d t}=-g_{m} V+g_{s}\left(V_{r e v}-V\right)
$$

(compare equation 2.8 with $V_{\text {rev }}<1$ ). We neglect the temporal fluctuations of the gating variable $s(t)$ here. This allows us to carry out an analytic calculation, and the result will confirm that the temporal fluctuations of 


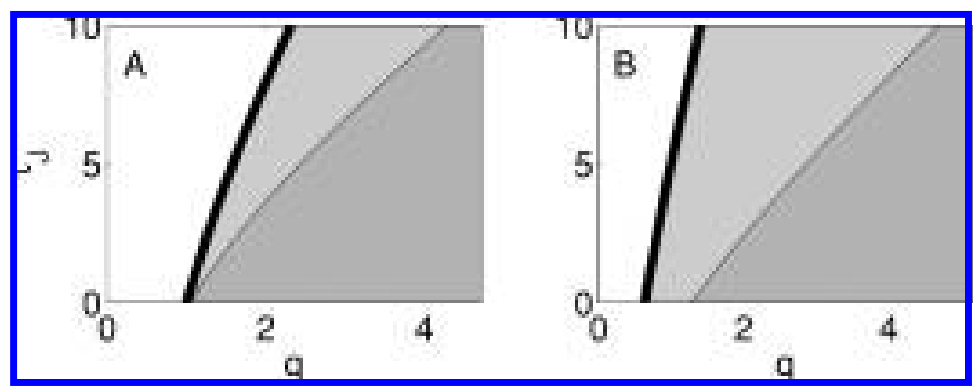

Figure 5: Injection of a square input pulse of total charge $q$ and duration $\tau_{J}$ into a neuron at rest elicits a spike if and only if $\left(q, \tau_{J}\right)$ lies in the shaded regions. With constant synaptic inhibition $\left(g_{s}=0.2\right)$ added, $\left(q, \tau_{J}\right)$ must lie in the dark shaded region for a spike to be elicited. (A) Linear integrate-and-fire neuron with $g_{m}=0.2, V_{\text {rev }}=-0.1$. (B) Theta neuron with $I=-0.1$. The boundaries are slanted to the right, so it requires less charge to elicit a spike with a brief pulse than with a broad one. This effect is greatly amplified by inhibition.

$s(t)$, while helpful (see section 3.3), are not needed for the suppression of the response to the less coherent distractor.

The extra term changes the resting potential from zero to $g_{s} V_{\text {rev }} /\left(g_{m}+g_{s}\right)$. Thus, we assume now that $V(0)=g_{s} V_{\text {rev }} /\left(g_{m}+g_{s}\right)$. As before, we add an input pulse during the time interval $\left[0, \tau_{J}\right]$ :

$$
\frac{d V}{d t}=-g_{m} V+g_{s}\left(V_{r e v}-V\right)+\frac{q}{\tau_{J}} \text { for } 0 \leq t \leq \tau_{J} .
$$

Again we ask whether the input pulse suffices to elicit a spike. The solution of equation 3.4 with $V(0)=g_{s} V_{\text {rev }} /\left(g_{m}+g_{s}\right)$ is

$$
V(t)=\frac{g_{s} V_{r e v}}{g_{m}+g_{s}} e^{-\left(g_{m}+g_{s}\right) t}+\frac{q / \tau_{J}+g_{s} V_{r e v}}{g_{m}+g_{s}}\left(1-e^{-\left(g_{m}+g_{s}\right) t}\right),
$$

so a spike is elicited if and only if

$$
\frac{g_{s} V_{\text {rev }}}{g_{m}+g_{s}} e^{-\left(g_{m}+g_{s}\right) \tau_{J}}+\frac{q / \tau_{J}+g_{s} V_{\text {rev }}}{g_{m}+g_{s}}\left(1-e^{-\left(g_{m}+g_{s}\right) \tau_{J}}\right) \geq 1,
$$

which is equivalent to

$$
q \geq \tau_{J} \frac{g_{m}+g_{s}-g_{s} V_{r e v}}{1-e^{-\left(g_{m}+g_{s}\right) \tau_{J}}}
$$


Inequality 3.5 defines the region of pairs $\left(q, \tau_{J}\right)$ for which a spike is elicited by the input pulse. This region is now dependent on $g_{m}, g_{s}$, and $V_{\text {rev }}$, and we therefore denote it by $\mathcal{S}_{g_{m}, g_{s}, V_{\text {rev }}}$. The dark shaded region in Figure $5 \mathrm{~A}$ represents $\mathcal{S}_{g_{m}, g_{s}, V_{\text {rev }}}$ with $g_{m}=0.2, g_{s}=0.2, V_{\text {rev }}=-0.1$. Notice that the boundary of the dark shaded region is slanted more strongly than the boundary of the union of the two shaded regions. This means that the amount of charge $q$ needed to elicit a spike depends more strongly on $\tau_{J}$ (that is, on coherence) in the presence of inhibition than in its absence.

If the reversal potential $V_{\text {rev }}$ were equal to the resting potential 0 , then inequality 3.5 would simply become

$$
q \geq \frac{\left(g_{m}+g_{s}\right) \tau_{J}}{1-e^{-\left(g_{m}+g_{s}\right) \tau_{J}}}
$$

Comparing inequalities 3.3 and 3.6, we see that in this case, inhibition simply has the effect of raising the effective leak conductances of the neuron from $g_{m}$ to $g_{m}+g_{s}$.

Interestingly, the advantage of briefer, stronger pulses over longer, weaker ones is in fact independent of the reversal potential $V_{\text {rev }}$. To see this, suppose that $\tau_{J}^{(1)}>\tau_{J}^{(2)}>0$. The minimum charge needed to elicit a spike is

$$
q_{\min }^{(1)}=\tau_{J}^{(1)} \frac{g_{m}+g_{s}-g_{s} V_{r e v}}{1-e^{-\left(g_{m}+g_{s}\right) \tau_{J}^{(1)}}}
$$

if $\tau_{J}=\tau_{J}^{(1)}$ and

$$
q_{\mathrm{min}}^{(2)}=\tau_{J}^{(2)} \frac{g_{m}+g_{s}-g_{s} V_{r e v}}{1-e^{-\left(g_{m}+g_{s}\right) \tau_{J}^{(2)}}}
$$

if $\tau_{J}=\tau_{J}^{(2)}$. The ratio $q_{\mathrm{min}}^{(1)} / q_{\mathrm{min}}^{(2)}$ can be thought of as the factor by which efficiency of the input pulse increases when its duration is decreased from $\tau_{J}^{(1)}$ to $\tau_{J}^{(2)}$. This ratio equals

$$
\frac{\tau_{J}^{(1)}}{\tau_{J}^{(2)}} \frac{1-e^{-\left(g_{m}+g_{s}\right) \tau_{J}^{(2)}}}{1-e^{-\left(g_{m}+g_{s}\right) \tau_{J}^{(1)}}}
$$

regardless of the value of $V_{\text {rev }}$.

3.2.3 Analysis for Theta Neurons. In this section, we sketch how the analysis of section 3.2.2 can be repeated for theta neurons; some additional details are provided in appendix B. We consider a theta neuron (see equation. 2.1) driven below threshold, that is, with $I<0$. We then add an input current 
$J=q / \tau_{J}$ during a time interval of duration $\tau_{J}$, and ask whether this current will elicit a spike. That is, we ask whether the solution $\theta$ of the initial value problem

$$
\begin{aligned}
\frac{d \theta}{d t} & =1-\cos \theta+\left(I+\frac{q}{\tau_{J}}\right)(1+\cos \theta) \\
\theta(0) & =\theta_{0}^{-}
\end{aligned}
$$

satisfies the inequality $\theta\left(\tau_{J}\right)>\theta_{0}^{+}$. Here $\theta_{0}^{ \pm}$denote the fixed points of the theta neuron (see equation 2.2). We recall that $\theta_{0}^{-}$is the stable fixed point and $\theta_{0}^{+}$the unstable one. We denote by $\mathcal{S}_{I}$ the set of pairs $\left(q, \tau_{J}\right)$ for which a spike is elicited. The union of the light and dark shaded regions in Figure 5B represents $\mathcal{S}_{I}$ for $I=-0.1$.

As in section 3.2.2, we now add constant synaptic inhibition to the theta neuron described by equation 2.1:

$$
\frac{d \theta}{d t}=1-\cos \theta+\left(I-\frac{3}{2} g_{s}\right)(1+\cos \theta)-g_{s} \sin \theta
$$

(compare equation 2.5). It is not hard to show (see appendix B) that equation 3.9 has a unique stable fixed point $\theta_{0, g_{s}}^{-} \in(-\pi, 0)$ and a unique unstable fixed point $\theta_{0, g_{s}}^{+} \in(0, \pi)$ for all $I$ and $g_{s}$ with $I-(3 / 2) g_{s}<0$. We then add again the input $J=q / \tau_{J}$ during a time interval of duration of $\tau_{J}$ and ask if it suffices to solicit a spike. Specifically, we consider the initial value problem:

$$
\begin{aligned}
\frac{d \theta}{d t} & =1-\cos \theta+\left(I+\frac{q}{\tau_{J}}-\frac{3}{2} g_{s}\right)(1+\cos \theta)-g_{s} \sin \theta \\
\theta(0) & =\theta_{0, g_{s}}^{-}
\end{aligned}
$$

We denote by $\mathcal{S}_{I, g_{s}}$ the region of pairs $\left(q, \tau_{J}\right)$ for which $\theta\left(\tau_{J}\right)>\theta_{0, g_{s}}^{+}$. To compute $\mathcal{S}_{I, g_{s}}$ numerically is easy. To compute it analytically is straightforward but tedious; we omit the details. The dark shaded portion of Figure 5B represents $\mathcal{S}_{I, g_{s}}$ for $I=-0.1$ and $g_{s}=0.2$. As before, inhibition causes the boundary of $\mathcal{S}$ to be much more slanted to the right; this demonstrates again that inhibition greatly amplifies the advantage of briefer, stronger input pulses over broader, weaker ones.

\subsection{The Timing of Inhibitory Pulses Lends an Advantage to the Input} That Entrains the Target. As noted earlier, entrainment of the target by a stimulus means, in all the experiments of this letter, that the target cells promptly respond with a spike to each pulse of the stimulus, but do not spike otherwise. This implies that inhibition is relatively weak when the 


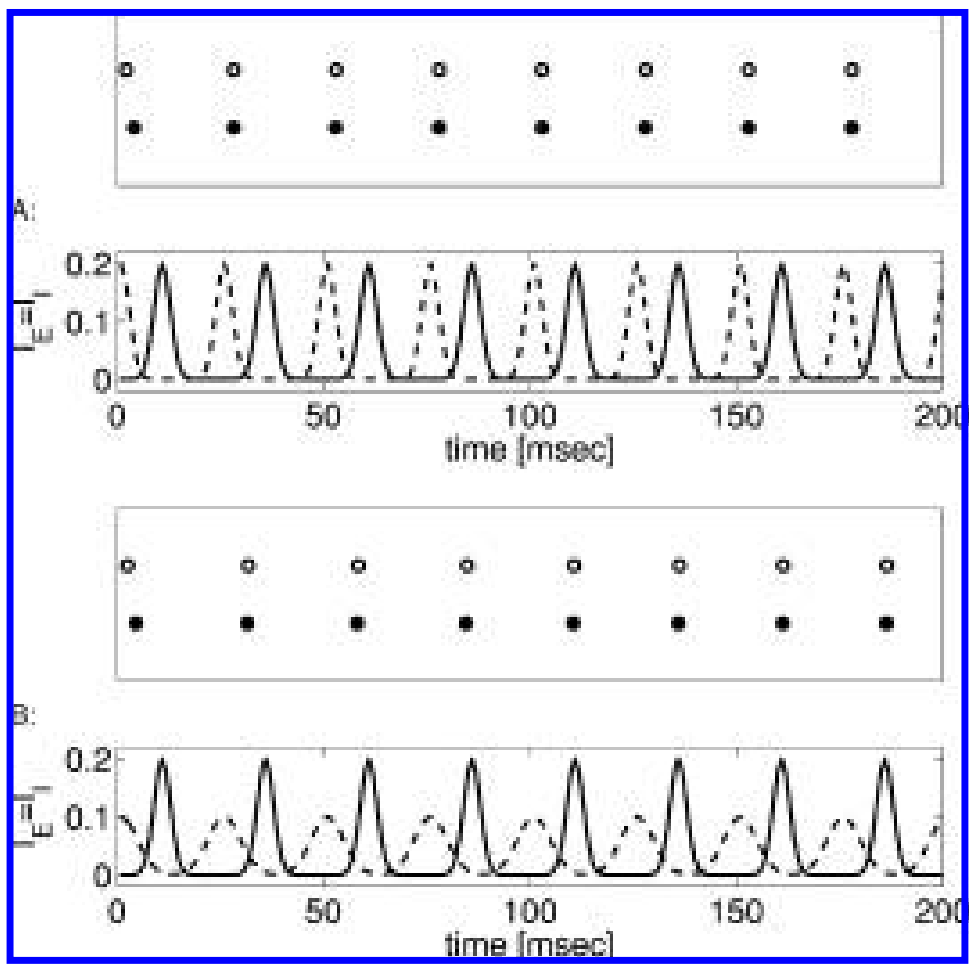

Figure 6: (A) A timing effect. Once input $B$ synchronizes with the target, input $A$ cannot break through even though its characteristics are identical with those of $B$ except for phase. (B) The timing effect of panel A disappears when input $B$ is made significantly less coherent than input $A$.

pulses of $A$ arrive and usually stronger when the pulses of $B$ arrive. ( $A$ raises inhibition immediately after it gives an input pulse to the target. This inhibition then decays, reaching its lowest value just before the arrival of the next input pulse from $A$.) Sometimes this effect alone determines which stimulus controls the target. Figure $6 \mathrm{~A}$ shows an example. The pulses of $B$ in this example are identical with those of $A$, but $A$ is phase delayed. The first pulse, at the beginning of the simulation, is a pulse of $B$, and this gives the input sequence $B$ the advantage over $A$. As a result, the target follows $B$ and ignores $A$. However, as Figure 6B illustrates, this timing effect disappears as soon as the distractor $B$ is somewhat less coherent than (but still as strong as) the primary stimulus $A$. We have also tried the experiment of Figure $6 \mathrm{~B}$ with other phase shifts and have found similar results.

We note that synchronization with the target would give the stimulus $A$ no advantage at all if there were no inhibition in the target network. In fact, 


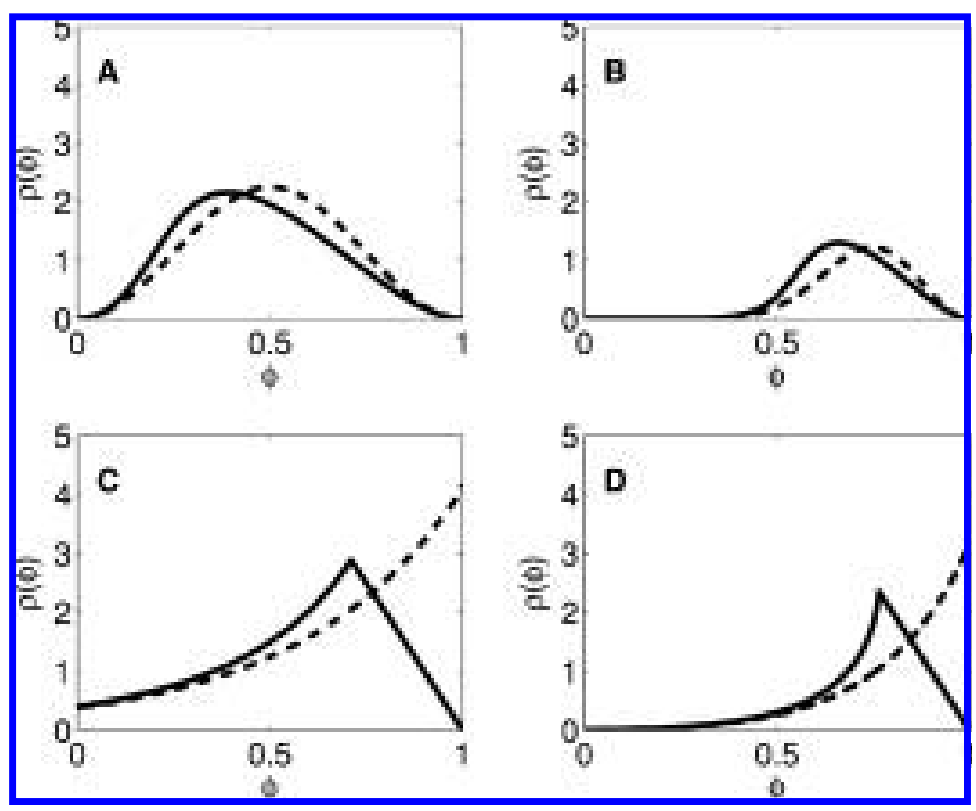

Figure 7: Phase response curves. A delta pulse of input of strength $\varepsilon$, finding its target at phase $\phi$, advances the phase of the target by $\varepsilon \rho(\phi)$. (A) Theta neuron, no inhibition, $I=0.02 ; \varepsilon=0.1$ (solid), and $\varepsilon \rightarrow 0$ (dashed). (B) Same as panel $\mathrm{A}$, but with a decaying inhibitory pulse added $\left(g=0.25, \tau_{I}=10\right)$. (C) Integrate-and-fire neuron, no inhibition, $g_{m}=0.1, I=0.11 ; \varepsilon=0.1$ (solid), and $\varepsilon \rightarrow 0$ (dashed). (D) Same as panel C, but with a decaying inhibitory pulse added $\left(g=0.25, \tau_{I}=10\right)$.

the noninfinitesimal phase response curve of a theta neuron has its peak in the earlier half of the period, and the infinitesimal phase response curve has its peak in the middle of the period (see appendix $C$ and Figure 7A). Inhibition shifts the peaks of the phase response curves to the right and creates a time interval at the beginning of the period during which the target is almost entirely input insensitive (see appendix $C$ and Figure 7B). So in the presence of local inhibition, the effectiveness of an excitatory input pulse is indeed greatest for pulses arriving near the end of the period. We note that the effect on the shape of the phase response curve of the theta neuron of synaptic inhibition resembles that of spike frequency adaptation (Gutkin, Ermentrout, \& Reyes, 2005, Figure 4B).

Of course, the discussion in the preceding paragraph depends on the neuronal model. For instance, for a linear integrate-and-fire neuron, the phase response curve peaks near the end of the period even in the absence of local inhibition (see appendix D and Figure 7C). As for the theta model, 


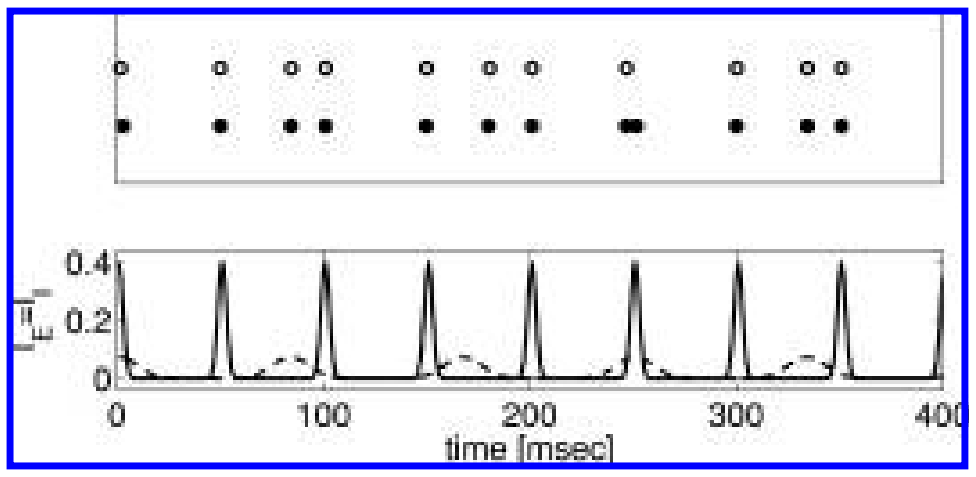

Figure 8: If $A$ oscillates far below gamma frequency, the distractor $B$ easily disrupts the rhythm in the target. Notice that this figure shows a $400 \mathrm{~ms}$ time window, whereas others in this letter show a $200 \mathrm{~ms}$ time window.

local inhibition shifts the peak of the phase response curve farther to the right and creates a time interval of input insensitivity near the beginning of the period (see appendix D and Figure 7D).

3.4 The Significance of the Gamma Frequency. The period of a gamma oscillation is the time that it takes for $\mathrm{GABA}_{\mathrm{A}}$-receptor-mediated inhibition to decay by approximately an order of magnitude. The mechanisms described here work only if the stimulus $A$ oscillates at least at a frequency in the gamma range. If the frequency is much lower, inhibition decays too much between the arrivals of the pulses of $A$. This is illustrated by Figure 8 , which shows the results of a simulation in which the coherent pulses of $A$ arrive at $20 \mathrm{~Hz}$ and the less coherent and weaker distractor $B$ oscillates at $12 \mathrm{~Hz}$. (As always, the parameter values are listed in appendix A.) The target is significantly affected by $B$.

3.5 Parameter Dependence. The numerical experiments that we have presented depend on many parameters, and it would be impossible to study the effects of varying these parameters simply by exhaustive search. However, we highlight the qualitative role played by the most important parameters here and demonstrate that stimulus selection based on gamma rhythmicity is a robust phenomenon.

3.5.1 Strength of Inhibition. Throughout this letter, we assume that the E- and I-cells receive equally strong inhibition: $g_{I E}=g_{I I}=g_{I}$. This assumption is natural because the distractor $B$ is assumed to target $E$ - and I-cells indiscriminately. If the distractor did not target the I-cells, then $g_{I I}$ could in fact be taken to be zero. 


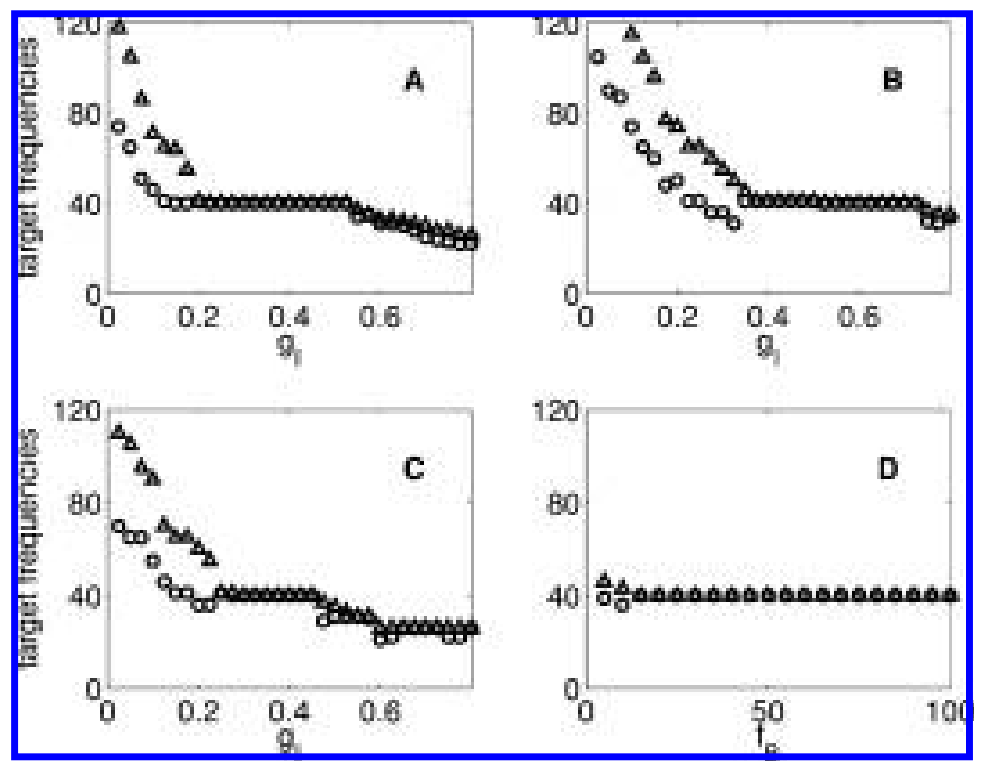

Figure 9: Parameter dependence of stimulus selection based on gamma rhythmicity. Frequencies of target E- and I-cells (circles and triangles) as functions of inhibitory conductance (A-C) and distractor frequency (D). (B) An example in which the distractor is far stronger on the average than the primary stimulus. (C) A case in which the coherence contrast between primary stimulus and distractor is weak. See the text for a more detailed discussion.

The inhibitory conductance $g_{I}$ has to be strong enough to protect the target against the distractor $B$ but not too strong to prevent its entrainment by $A$. To show that there is a broad range of values $g_{I}$ satisfying both constraints, we repeat the experiment of Figure $3 \mathrm{~A}$ varying $g_{I}$, with all other parameters fixed as in Figure 3A. Figure 9A shows the frequencies of the Eand I-cells of the target (indicated as circles and triangles, respectively) as functions of $g_{I}$. For $g_{I} \in[0.2,0.525]$, we see a plateau representing entrainment by stimulus A: both target cells spike at $40 \mathrm{~Hz}$. We now consider how the location and width of this plateau change as the distractor strength and coherence are varied.

3.5.2 Distractor Strength. Changes in the distractor strength tend to shift the plateau of entrainment seen in Figure 9A without affecting its width very much. In Figure 9B, we show the same experiment as in Figure 9A but with the distractor strength doubled: $C_{B}=Q_{B}=0.12$. Since $C_{A}=0.04$, this means that stimulus $B$ is now, on the average, three times stronger than stimulus $A$. Nevertheless, the more coherent gamma frequency stimulus $A$ still prevails over a wide range of values of $g_{I}$. In the experiments of this 
letter, the temporally averaged strength of the stimulus $A$ is not larger (and sometimes smaller) than that of the distractor $B$. If $A$ were not just more coherent than $B$ but also of greater average strength, the range of values of $g_{I}$ for which the target is entrained by $A$ would broaden.

3.5.3 Distractor Coherence. Even when the contrast in coherence between $A$ and $B$ is relatively modest, there is a range of values of $g_{I}$ in which stimulus $A$ prevails. Figure $9 \mathrm{C}$ demonstrates this. Here the parameters are again chosen as in Figure 3A, but $\sigma_{B}$ has been lowered to 5 (earlier it was 9). The plateau of $40 \mathrm{~Hz}$ entrainment is narrower than before. There is now also a range of values of $g_{I}$ for which the target is entrained to $B$ at $25 \mathrm{~Hz}$.

3.5.4 Distractor Frequency. The frequency $f_{B}$ of the less coherent, distracting stimulus $B$ is largely irrelevant. To demonstrate this, we begin again with the experiment of Figure 3A but raise the value of $g_{I}$ from 0.2 to 0.35 . (Figure 9A indicates that 0.2 lies near the lower edge of the range of values of $g_{I}$ in which $A$ entrains the target; 0.35 is much closer to the center of that range.) We vary the distractor frequency. Figure 9D shows the result, confirming the irrelevance of $f_{B}$ over a wide range. To understand why entrainment fails when $f_{B}$ is very low, note that the temporal average of $B$ is fixed here; thus, when the pulses of $B$ are very infrequent, they are also very strong-strong enough to distract the target. Not surprisingly, this effect becomes more pronounced when $g_{I}$ is lowered (when $g_{I}=0.2$, entrainment occurs only when $f_{B} \geq 25$ ) but disappears when the distractor strength is lowered (data not shown).

We remark that the assumption $f_{B}<f_{A}$, although not needed in our study, would in fact seem natural here. The abstract networks discussed here should be thought of as models of what happens at higher levels of processing; it seems reasonable to conjecture that at those levels, "distractor" input might arrive not only with less coherence but in fact with less drive than attended input. Lower drive typically translates into (at least somewhat) lower oscillation frequency (see, e.g., Figure 3C of White, Banks, Pearce, \& Kopell, 2000, or Figure 2 of Börgers \& Kopell, 2005).

3.5.5 Heterogeneity and Noise. Our experience suggests that heterogeneities in neuronal and network properties or noisy external drives do not prevent stimulus selection based on gamma rhythmicity and in fact may facilitate it in some cases. We have no analysis explaining the latter observation but present a sample simulation in Figure 10. The simulation underlying Figure 10 involves a target of 80 E-cells and 20 I-cells. Parameters are essentially those of Figure 3A; however, $g_{I}$ has been reduced from 0.2 to 0.1 (bringing it clearly below the range of entrainment according to Figure 9A), and heterogeneity and noise in external drives and network connectivity have been introduced (see appendix A for details). Figure 10 shows that the target is noisily but unmistakably entrained by the $40 \mathrm{~Hz}$ 


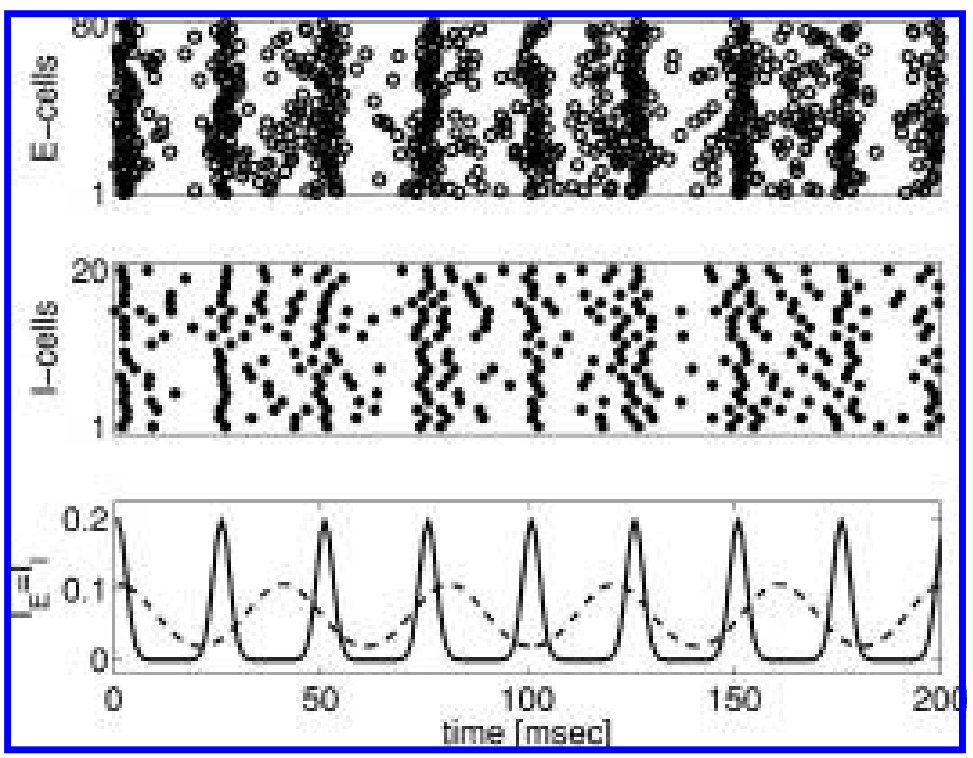

Figure 10: A noisy version of Figure 3A with reduced inhibitory conductance.

stimulus $A$. In the simulation underlying Figure 10, the average strength of input A varies from cell to cell; the solid curve in the bottom panel of Figure 10 indicates the average of input A over all cells of the network.

\section{An Incoherent Distractor Can Promote Its Own Suppression}

Suppose that the primary stimulus $A$, presented alone, activates the E-cell but is insufficient to activate the I-cells. In a real network, the I-cells might, for instance, be of type II (Tateno, Harsch, \& Robinson, 2004), and stimulus $A$ alone might not suffice to bring them above their spiking threshold. In our model network of two theta neurons, we make the I-cell unresponsive by injecting a strong, constant, hyperpolarizing current. Figure 11A shows the result of such a simulation. Since the I-cell does not spike, the E-cell is left vulnerable to distracting stimuli. Figure 11B shows results of a simulation illustrating this point; here, the distracting input was given to the E-cell only. If the distractor $B$ also drives the I-cell, it may help activate the I-cell and thereby restore entrainment of the target network by $A$. This is illustrated by the simulation result shown in Figure 11C. (Parameter values for Figure 11 are given in appendix A.) We remark that the oscillatory nature of the distractor is irrelevant here; the essential point is that the distractor activates the I-cell enough to enable it to participate. 


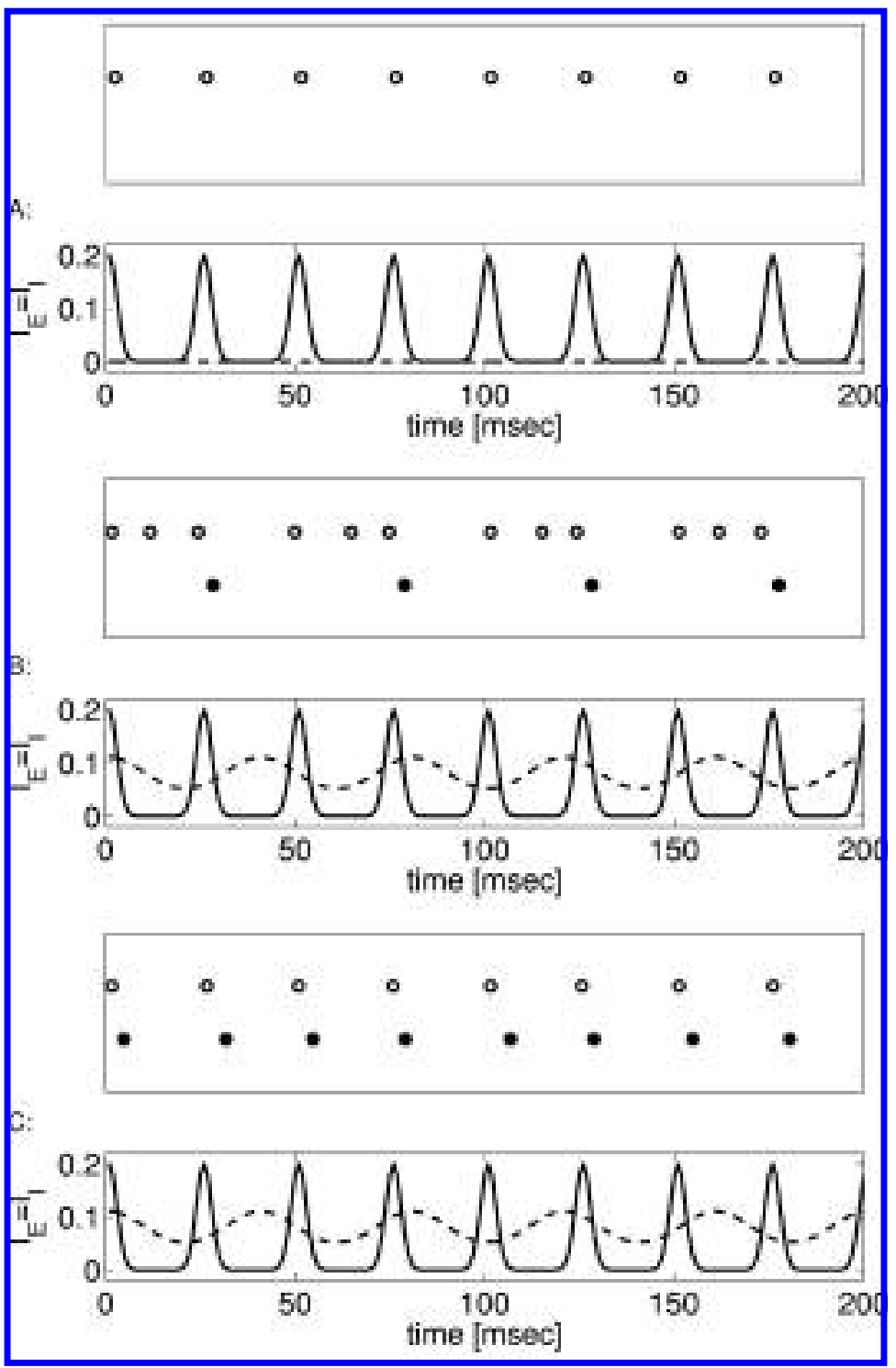

Figure 11: When the primary stimulus $A$ does not suffice to activate the I-cell (A), the E-cell is left vulnerable to distractors (B). However, a distracting stimulus $B$ to the I-cell may activate that cell, and thereby enable entrainment of the target to stimulus $A(\mathrm{C})$. 
We note that an alternative way of overcoming hyperpolarization of the I-cells, enabling them to fire and thereby invoking the mechanism that shuts out distractors, would be the selective enhancement of the coherence of the primary input stream $A$ to the I-cells of the target network.

\section{Discussion}

Several possible functional roles of neuronal coherence in the brain have been suggested in the literature. Coherence strengthens the effectiveness of excitatory signals (Murthy \& Fetz, 1994; Singer, 1999), weakens the effectiveness of inhibitory signals by leaving windows of opportunity (Börgers, Epstein, \& Kopell, 2005; Börgers \& Kopell, 2005; Lumer, 2000; Tiesinga, 2002; Tiesinga, Fellous, Salinas, José, \& Sejnowski, 2004), may play an important role in the creation of cell assemblies (Olufsen, Whittington, Camperi, \& Kopell, 2003), and may be the key to solving the "binding problem" Engel, Fries, \& Singer, 2001; Singer \& Gray, 1995; von der Malsburg \& Schneider, 1986). In this letter, we have demonstrated that gamma rhythms may also facilitate stimulus selection. When excitatory input to an E/I network (not just to a single target cell) comes in the form of a sequence of coherent pulses in the gamma frequency range, downstream response to less coherent competitors is suppressed.

Fries (2005) suggested that timing is crucial: the phase dependence of the input sensitivity of the target lends an advantage to well-timed inputs over ill-timed ones. In our model, timing does play a role when there is strong inhibition in the target, as shown, for instance, in Figure 6A, but not otherwise (see Figure 4 and our discussion of phase response curves). In our numerical experiments, timing effects can tip the balance when the stimuli are close to each other-or identical, as in Figure 6A. There is, however, a second important effect: inhibition in the target network, by itself and independent of timing, greatly amplifies the advantage of coherent inputs over incoherent ones (see e.g., Figure 6B). Once an oscillatory input entrains the target, the phase difference between it and the target is dictated by the dynamics; indeed this phase difference is favorable to the entraining input.

In Börgers et al. (2005), we proposed a different way in which gamma rhythmicity may play a role in stimulus selection; there we showed that gamma rhythmicity helps a strongly driven assembly suppress activity in a less strongly driven one if the two assemblies share a single interneuron network. Thus, the competition was between two assemblies in Börgers et al. (2005), whereas here it is between two stimuli competing for control over a single assembly. Combinations of the two scenarios are possible and plausible; for instance, two different stimuli might drive two different but overlapping assemblies within a larger network.

We have assumed purely excitatory input to a target network that includes local recurrent inhibition. This is in contrast with other recent models 
(Tiesinga, 2005; Mishra, Fellous, \& Sejnowski, 2006) in which excitatory and inhibitory input streams drive a single neuron, with possible attentional modulation of coherence and phase of the two streams independently.

In our model, the entire target network is entrained by the primary input $A$, and therefore frequency and phase of the response are the only degrees of freedom available to encode stimulus identity and intensity. In a larger, more realistic version of our model, inputs $A$ and $B$ would naturally stimulate different ensembles of neurons; the overlap of the two ensembles would then be the analog of the target network in this study. Furthermore, the spiking frequency of individual cells would not necessarily have to be identical with the population frequency in such a model. Thus, additional degrees of freedom - the set of neurons responding and the mean frequency of individual cells-become available to encode stimulus identity and intensity when the model is made larger and more realistic. How the results of this study extend to such more complex situations will be a subject of future work.

We did not allow feedback from target to upstream sources in this study. It remains to be investigated how such feedback, which, of course, is typically present in the brain, affects the mechanisms discussed here.

Codes used to produce the numerical results of this letter can be obtained by sending an e-mail request to christoph.borgers@tufts.edu.

\section{Appendix A: Parameter Values Used in Simulations}

For Figure 2:

$$
\begin{gathered}
g_{E E}=0 \quad g_{E I}=0.05 \quad g_{I}=0.2 \\
C_{A}=0.04 \quad Q_{A}=0.04 \quad f_{A}=40 \quad \sigma_{A}=2 \quad \varphi_{A}=0 \\
C_{B}=0 \quad Q_{B}=0 \quad \varphi_{B}=0
\end{gathered}
$$

We have omitted the indices $i$ labeling neurons because there is only one cell of each kind, $E$ and $I$. We have also omitted the indices $E$ and $I$ because the two cells receive the same input stream here.

For Figure 3A:

$$
\begin{array}{ccccc}
g_{E E}=0 \quad g_{E I}=0.05 & g_{I}=0.2 \\
C_{A}=0.04 & Q_{A}=0.04 & f_{A}=40 & \sigma_{A}=2 & \varphi_{A}=0 \\
C_{B}=0.06 & Q_{B}=0.06 & f_{B}=25 & \sigma_{B}=9 & \varphi_{B}=0
\end{array}
$$

For Figure 3B:

$$
\begin{gathered}
g_{E E}=0 \quad g_{E I}=0.05 \quad g_{I}=0.2 \\
C_{A}=0.04 \quad Q_{A}=0.04 \quad f_{A}=40 \quad \sigma_{A}=2 \quad \varphi_{A}=0 \\
C_{B}=0.06 \quad Q_{B}=20 \quad f_{B}=65 \quad \sigma_{B}=9 \quad \varphi_{B}=0
\end{gathered}
$$


The ratio $T_{B} / \sigma_{B}=1000 /\left(f_{B} \sigma_{B}\right)$ is smaller here than elsewhere in this letter. This is the reason that $Q_{B}$ is taken to be so large here (see the paragraph following equation 2.6). The choice $Q_{B}=20$ yields a sizable but not overwhelming oscillatory component, as shown in Figure 3B.

For Figure 3C:

$$
\begin{array}{ccccc}
g_{E E}=0 & g_{E I}=0.05 & g_{I}=0.5 \\
C_{A}=0.04 & Q_{A}=0.04 & f_{A}=40 & \sigma_{A}=2 & \varphi_{A}=0 \\
C_{B}=0.11 & Q_{B}=0.15 & f_{B}=25 & \sigma_{B}=9 & \varphi_{B}=0
\end{array}
$$

For Figure 6A:

$$
\begin{array}{ccccc}
g_{E E}=0 & g_{E I}=0.05 & g_{I}=0.2 & \\
C_{A}=0.04 & Q_{A}=0.04 & f_{A}=40 & \sigma_{A}=2 & \varphi_{A}=0.4 \\
C_{B}=0.04 & Q_{B}=0.04 & f_{B}=40 & \sigma_{B}=2 & \varphi_{B}=0
\end{array}
$$

For Figure 6B, the parameters are as in Figure 6A, but with $\sigma_{B}=4$ in place of $\sigma_{B}=2$.

For Figure 8:

$$
\begin{array}{ccccc}
g_{E E}=0 & g_{E I}=0.05 & g_{I}=0.2 \\
C_{A}=0.04 & Q_{A}=0.04 & f_{A}=20 & \sigma_{A}=2 & \varphi_{A}=0 \\
C_{B}=0.02 & Q_{B}=0.02 & f_{B}=12 & \sigma_{B}=9 & \varphi_{B}=0
\end{array}
$$

For Figure 10:

$$
\begin{gathered}
g_{E E}=0 \quad g_{E I}=0.05 \quad g_{I}=0.1 \\
C_{A, E, i}=0.04+0.04 Z_{E, i} \quad C_{A, I, i}=0.04+0.04 Z_{I, i} \\
Q_{A}=0.04 \quad f_{A}=40 \quad \sigma_{A}=2
\end{gathered}
$$

$Z_{E, i}\left(1 \leq i \leq N_{E}\right)$ and $Z_{I, i}\left(1 \leq i \leq N_{I}\right)$ are independent standard gaussians.

$$
C_{B}=0.06 \quad Q_{B}=0.06 \quad f_{B}=25 \quad \sigma_{B}=9
$$

In addition, every synaptic connection is removed with probability $1 / 2$ and doubled in strength if not removed, and in every time step, the random term $0.2 \sqrt{\Delta t} Z$ is added to $\theta$ for each neuron, where $Z$ is a standard gaussian. 
For Figure 11A:

$$
\begin{gathered}
g_{E E}=0 \quad g_{E I}=0.05 \quad g_{I}=0.2 \\
C_{A}=0.04 \quad Q_{A}=0.04 \quad f_{A}=40 \quad \sigma_{A}=2 \\
C_{B}=Q_{B}=0
\end{gathered}
$$

In addition, the I-cell receives a constant hyperpolarizing current equal to -0.3 .

For Figure 11B, parameters are as in Figure 11A, but the E-cell receives an additional distracting input:

$$
\begin{gathered}
C_{B, E}=0.08 \quad Q_{B, E}=0.04 \quad f_{B}=25 \quad \sigma_{B}=9 \\
C_{B, I}=Q_{B, I}=0
\end{gathered}
$$

For Figure 11C, parameters are as in Figure 11B, but the I-cell receives the same distracting input as the E-cell.

\section{Appendix B: Details of the Analysis for Theta Neurons}

The Region $\mathcal{S}_{I}$. The region $\mathcal{S}_{I}$ is the set of pairs $\left(q, \tau_{J}\right)$ for which the solution $\theta$ of equations. 3.7 and 3.8 satisfies the inequality $\theta\left(\tau_{J}\right)>\theta_{0}^{+}$. The fixed points $\theta_{0}^{-}$and $\theta_{0}^{+}$are given by equation 2.2. It is clear that $\theta\left(\tau_{J}\right)$ cannot be greater than $\theta_{0}^{+}$unless $I+q / \tau_{J}>0$. The time that it takes for $\theta$ to increase from $\theta_{0}^{-}$to $\theta_{0}^{+}$then equals

$$
\begin{aligned}
& \int_{\theta_{0}^{-}}^{\theta_{0}^{+}} \frac{d t}{d \theta} d \theta=\int_{\theta_{0}^{-}}^{\theta_{0}^{+}} \frac{1}{1-\cos \theta+\left(I+q / \tau_{J}\right)(1+\cos \theta)} d \theta \\
& =\frac{1}{\sqrt{I+q / \tau_{J}}}\left[\arctan \frac{\tan (\theta / 2)}{\sqrt{I+q / \tau_{J}}}\right]_{\theta_{0}^{-}}^{\theta_{0}^{+}}=\frac{2}{\sqrt{I+q / \tau_{J}}} \arctan \frac{\tan \left(\theta_{0}^{+} / 2\right)}{\sqrt{I+q / \tau_{J}}} \\
& =\frac{2}{\sqrt{I+q / \tau_{J}}} \arctan \frac{\tan \arccos (1 / \sqrt{1-I})}{\sqrt{I+q / \tau_{J}}}=\frac{2}{\sqrt{I+q / \tau_{J}}} \arctan \frac{\sqrt{-I}}{\sqrt{I+q / \tau_{J}}} .
\end{aligned}
$$

The region $\mathcal{S}_{I}$ is therefore given by the inequality

$$
\frac{2}{\sqrt{I+q / \tau_{J}}} \arctan \frac{\sqrt{-I}}{\sqrt{I+q / \tau_{J}}}<\tau_{J},
$$


and its boundary, the bold line in Figure 5B, is characterized by the equation

$$
\frac{2}{\sqrt{I+q / \tau_{J}}} \arctan \frac{\sqrt{-I}}{\sqrt{I+q / \tau_{J}}}=\tau_{J}
$$

Equation B.1 is not an explicit description of the boundary

$$
q=q\left(\tau_{J}\right)
$$

of the shaded reason (the bold line) in Figure 5B. However, it is clear that for any fixed $\tau_{J}>0$ and $I<0$, the equation has a unique solution $q$ with $I+q / \tau_{J}>0$, since the left-hand side, viewed as a function of $q$, decreases monotonically from $\infty$ to 0 as $q$ increases from $-\tau_{J} I$ to $\infty$. We note that equation B.1 implies $q\left(\tau_{J}\right) / \tau_{J} \rightarrow \infty$ as $\tau_{J} \rightarrow 0$, a fact that will be used shortly.

Numerically, it is easy to solve equation B.1 for $q$ in terms of $\tau_{J}$. Furthermore, we can derive the behavior of the boundary for small $\tau_{J}$ explicitly from equation B.1. Using the Taylor expansion

$$
\arctan x=x-\frac{x^{3}}{3}+O\left(x^{5}\right) \quad \text { as } x \rightarrow 0
$$

equation B.1 can be written as follows in the limit as $\tau_{J} \rightarrow 0$ :

$$
\frac{2}{\sqrt{I+q / \tau_{J}}}\left[\frac{\sqrt{-I}}{\sqrt{I+q / \tau_{J}}}-\frac{(-I)^{3 / 2}}{3\left(I+q / \tau_{J}\right)^{3 / 2}}+O\left(\tau_{J}^{5 / 2}\right)\right]=\tau_{J}
$$

and therefore

$$
\frac{2 \sqrt{-I}}{q+\tau_{J} I}+\frac{2 I \sqrt{-I}}{3\left(q+\tau_{J} I\right)^{2}} \tau_{J}=1+o\left(\tau_{J}^{3 / 2}\right)
$$

(The $O(\cdot)$ in equation B.2 turned into $o(\cdot)$ in equation B.3 because $q / \tau_{J} \rightarrow \infty$ as $\tau_{J} \rightarrow 0$, a fact noted earlier.)

Equation B.3 is a quadratic equation for $q+\tau_{J} I$, with solutions

$$
q_{ \pm}+\tau_{J} I=\sqrt{-I} \pm \sqrt{-I+\frac{2}{3} I \sqrt{-I} \tau_{J}}+o\left(\tau_{J}^{3 / 2}\right)
$$

Expanding the right-hand side around $\tau_{J}=0$, we find

$$
q_{ \pm}=\sqrt{-I} \pm\left(\sqrt{-I}+\frac{1}{3} \tau_{J} I\right)-\tau_{J} I+o\left(\tau_{J}^{3 / 2}\right)
$$


The solution $q_{-}$is spurious: it violates the condition $q / \tau_{J} \rightarrow \infty$ as $\tau_{J} \rightarrow 0$, which was used in our expansion. Thus, $q_{+}$is the relevant solution, and the boundary of $\mathcal{S}_{I}$ is given by

$$
q \sim 2 \sqrt{-I}+\frac{2}{3} \tau_{J}|I|
$$

as $\tau_{J} \rightarrow 0$. Equation B.4 describes the tangent line to the boundary of $\mathcal{S}_{I}$ at $q=2 \sqrt{-I}, \tau_{J}=0$. However, the segment of the boundary of $\mathcal{S}_{I}$ shown as a bold line in Figure 5B is nearly straight, and equation B.4 therefore in fact describes it with good accuracy throughout.

Equation B.4 shows that the amount of charge needed to elicit a spike rises like $(2 / 3) \tau_{J}|I|$ as $\tau_{J}$ increases. Thus, the advantage of a briefer, stronger pulse over a broader, weaker one rises as $|I|$ rises: the more strongly hyperpolarized the target neuron, the greater is the advantage of coherent inputs over incoherent ones.

Fixed Points of Equation 3.9. We will prove that for $I-(3 / 2) g_{s}<0$, there are exactly two fixed points of equation 3.9 in $[-\pi, \pi]$ : a stable one in $(-\pi, 0)$ and an unstable one in $(0, \pi)$. Fixed points of equation 3.9 are solutions of $F(\theta)=0$ with

$$
F(\theta)=1-\cos \theta+\left(I-\frac{3}{2} g_{s}\right)(1+\cos \theta)-g_{s} \sin \theta
$$

First, we note that

$$
F(-\pi)=2>0, \quad F(0)=2\left(I-\frac{3}{2} g_{s}\right)<0, \quad \text { and } \quad F(\pi)=2>0 .
$$

So in particular, if $\theta \in[-\pi, \pi]$ is a solution of $F(\theta)=0$, then $\sin \theta \neq 0$. This implies that no two solutions $\theta \in[-\pi, \pi]$ of $F(\theta)=0$ can be negatives of each other, since

$$
F(\theta)=0 \Rightarrow F(-\theta)=F(\theta)+2 g_{s} \sin \theta=2 g_{s} \sin \theta \neq 0 .
$$

The inequalities B.5 imply that there are at least one stable fixed point in $(-\pi, 0)$, and one unstable fixed point in $(0, \pi)$. We now show that there can be no more than two fixed points in $[-\pi, \pi]$. Suppose there were three different fixed points $\theta_{i} \in[-\pi, \pi], i=1,2,3$. Since no two of these can be negatives of each others, their cosines,

$$
x_{i}=\cos \theta_{i}, \quad i=1,2,3,
$$


would have to be different from each other. But

$$
\begin{aligned}
F\left(\theta_{i}\right)=0 & \Rightarrow 1-\cos \theta_{i}+\left(I-\frac{3}{2} g_{s}\right)\left(1+\cos \theta_{i}\right)=g_{s} \sin \theta_{i} \\
& \Rightarrow\left(1-\cos \theta_{i}+\left(I-\frac{3}{2} g_{s}\right)\left(1+\cos \theta_{i}\right)\right)^{2}=g_{s}^{2}\left(1-\cos ^{2} \theta_{i}\right) \\
& \Rightarrow\left(1-x_{i}+\left(I-\frac{3}{2} g_{s}\right)\left(1+x_{i}\right)\right)^{2}=g_{s}^{2}\left(1-x_{i}^{2}\right)
\end{aligned}
$$

This is a contradiction, since $x_{1}, x_{2}$, and $x_{3}$ would now be three different solutions of the quadratic equation B.6.

\section{Appendix C: Phase Response Curve of a Theta Neuron with Decaying Inhibition}

In this appendix, we briefly review the standard phase response function for the theta neuron (Ermentrout, 1996). We then define and compute a phase response function for a theta neuron under the influence of exponentially decaying inhibition.

Consider the theta neuron given by equation 2.1 and assume $I>0$. The "phase" $\phi \in[0,1]$ corresponding to $\theta \in[-\pi, \pi]$ is the time that it takes to advance from $-\pi$ to $\theta$, divided by the period $T=\pi / \sqrt{I}$. If a delta pulse of strength $\varepsilon>0$ is added to $I$ when the neuron is at phase $\phi$, its phase advances by an amount denoted by $\varepsilon \rho(\phi)$. A standard and elementary calculation yields

$$
\rho(\phi)=\frac{1}{\varepsilon}\left[\frac{1}{2}+\frac{1}{\pi} \tan ^{-1}\left[\tan \left(\pi\left(\phi-\frac{1}{2}\right)\right)+\frac{\varepsilon}{\sqrt{I}}\right]-\phi\right] .
$$

We refer to $\rho$ as the phase response function of the theta neuron. Its graph is called the phase response curve. Passing to the limit as $\varepsilon \rightarrow 0$, one obtains the infinitesimal phase response function

$$
\rho_{0}(\phi)=\frac{1}{\pi \sqrt{I}} \cos ^{2}\left(\pi\left(\phi-\frac{1}{2}\right)\right) .
$$

Its graph is called the infinitesimal phase response curve. Figure 7A shows the graph of $\rho$ for $I=0.02, \varepsilon=0.1$ (solid), and the graph of $\rho_{0}$ for $I=0.02$ (dashed). 
Next we consider a theta neuron subject to an exponentially decaying pulse of synaptic inhibition arriving at time $t=0$ (see equation 2.5):

$$
\frac{d \theta}{d t}=1-\cos \theta+\left(I-\frac{3}{2} g e^{-t / \tau_{I}}\right)(1+\cos \theta)-g e^{-t / \tau_{I}} \sin \theta
$$

with $I>0, g>0$, and $\tau_{I}=10$. The letter $T$ now denotes the time that it takes for a solution $\theta$ of equation C. 1 to advance from $-\pi$ to $\pi$. The phase $\phi \in[0,1]$ corresponding to $\theta \in[-\pi, \pi]$ is again the time that it takes for a solution of equation C.1 to advance from $-\pi$ to $\theta$, divided by $T$. If a delta pulse of strength $\varepsilon>0$ is added to $I$ at time $\phi T$, the time that it takes for $\theta$ to reach $\pi$ is shortened to $\hat{T}<T$. The phase response curve is the graph of

$$
\rho(\phi)=\frac{1}{\varepsilon} \frac{T-\hat{T}}{T}
$$

As before, the infinitesimal phase response function $\rho_{0}$ is obtained by passing to the limit $\varepsilon \rightarrow 0$. We have no analytic expression for $\rho$ and $\rho_{0}$, but it is straightforward to calculate these curves numerically for given parameter values. Figure 7B shows the graphs of $\rho$ for $I=0.02, g=0.25, \varepsilon=0.1$ (solid), and $\rho_{0}$ for $I=0.02, g=0.25$ (dashes).

\section{Appendix D: Phase Response Curve of an Integrate-and-Fire Neuron with Decaying Inhibition}

Since some of the analysis of this letter is based on integrate-and-fire neurons, we repeat the calculations of appendix $\mathrm{C}$ for this model. We consider equation 2.7 with the threshold potential $V_{T}=1$ and the reset potential $V_{R}=0$. We assume that the drive is above the spiking threshold, that is, $I>g_{m}$. The "phase" $\phi \in[0,1]$ corresponding to $V \in[0,1]$ is the time that it takes for the membrane potential to advance from 0 to $V$, divided by the period

$$
T=\frac{1}{g_{m}} \ln \frac{I}{I-g_{m}}
$$

When an integrate-and-fire neuron receives a delta pulse of current input of strength $\varepsilon>0$, its membrane potential is assumed to rise instantaneously from its value $V$ just prior to the arrival of the pulse to

$$
V_{+}=\left\{\begin{array}{cl}
V+\varepsilon & \text { if } V+\varepsilon \leq 1 \\
1 & \text { otherwise }
\end{array}\right.
$$


This raises the phase from $\phi$ to a new value $\phi_{+}$. The phase response function $\rho$ is defined by the equation

$$
\varepsilon \rho(\phi)=\phi_{+}-\phi
$$

A straightforward calculation yields

$$
\rho(\phi)=\frac{\min (z, 1)-\phi}{\varepsilon} \quad \text { with } \quad z=\frac{\ln \left(\left(1-g_{m} / I\right)^{\phi}-\varepsilon_{m} / I\right)}{\ln \left(1-g_{m} / I\right)} .
$$

The limit of $\rho$ as $\varepsilon \rightarrow 0$ is the infinitesimal phase response function, denoted by $\rho_{0}$; it is not hard to show that

$$
\rho_{0}(\phi)=\frac{g_{m} / I}{\left(1-g_{m} / I\right)^{\phi} \ln \left(1 /\left(1-g_{m} / I\right)\right)} .
$$

Figure 7C shows the graph of $\rho$ for $g_{m}=0.1, I=0.11$ and $\varepsilon=0.1$ (solid) and the graph of $\rho_{0}$ for $g_{m}=0.1$ and $I=0.11$ (dashed).

Next we consider an integrate-and-fire neuron, driven above the spiking threshold, subject to an exponentially decaying pulse of synaptic inhibition arriving at time $t=0$ :

$$
\frac{d V}{d t}=-g_{m} V+I-g e^{-t / \tau_{I}} V
$$

with $I>g_{m}, g>0$, and $\tau_{I}=10$. The letter $T$ now denotes the time that it takes for a solution $V$ of equation D.1 to advance from 0 to 1 . The phase $\phi \in[0,1]$ corresponding to $V \in[-1,1]$ is again the time that it takes for a solution of equation D.1 to advance from 0 to $V$, divided by $T$. If a delta pulse of strength $\varepsilon>0$ is added to $I$ at time $\phi T$, the time that it takes for $V$ to reach 1 is shortened to $\hat{T}<T$. The phase response curve is the graph of

$$
\rho(\phi)=\frac{1}{\varepsilon} \frac{T-\hat{T}}{T}
$$

As before, the infinitesimal phase response curve $\rho_{0}$ is obtained by passing to the limit $\varepsilon \rightarrow 0$. We have no analytic expression for $\rho$ and $\rho_{0}$, but it is straightforward to calculate these curves numerically for given parameter values. Figure 7D shows the graphs of $\rho$ for $g_{m}=0.1, I=0.11, g=0.25$, $\varepsilon=0.1$ (solid), and $\rho_{0}$ for $g_{m}=0.1, I=0.11, g=0.25$ (dashed).

\section{Acknowledgments}

We thank the anonymous referees for their valuable suggestions, which led to substantial improvements of the manuscript. This work was supported by NSF grants DMS-0418832 (to C.B.) and DMS-0211505 (to N.J.K.) and by 
NIH grant R01 NS-46058 (to N.J.K.) as part of the NSF/NIH Collaborative Research in Computational Neuroscience Program.

\section{References}

Abbott, L. F. (1999). Lapicque's introduction of the integrate-and-fire model neuron (1907). Brain Res. Bull., 50(5/6), 303-304.

Börgers, C., Epstein, S., \& Kopell, N. (2005). Background gamma rhythmicity and attention in cortical local circuits: A computational study. Proc. Natl. Acad. Sci. USA, 102(19), 7002-7007.

Börgers, C., \& Kopell, N. (2005). Effects of noisy drive on rhythms in networks of excitatory and inhibitory neurons. Neural Comp., 17(3), 557-608.

Engel, A. K., Fries, P., \& Singer, W. (2001). Dynamic predictions: Oscillations and synchrony in top-down processing. Nat. Rev. Neurosci., 2, 704-716.

Ermentrout, G. B. (1996). Type I membranes, phase resetting curves, and synchrony. Neural Comp., 8, 879-1001.

Ermentrout, G. B., \& Kopell, N. (1986). Parabolic bursting in an excitable system coupled with a slow oscillation. SIAM I. Appl. Math., 46, 233-253.

Fries, P. (2005). A mechanism for cognitive dynamics: Neuronal communication through neuronal coherence. Trends in Coonitive Sciences, 9, 474-480.

Fries, P., Schröder, J.-H., Roelfsema, P. R., Singer, W., \& Engel, A. K. (2002). Oscillatory neuronal synchronization in primary visual cortex as a correlate of stimulus selection. J. Neurosci., 22, 3739-3754.

Funabiki, K., Koyano, K., \& Ohmori, H. (1998). The role of GABaergic inputs for CD in the neurones of nucleus laminaris of the chick. I. Physiol. (London), 508, 851-869.

Grande, L. A., Kinney, G. A., Miracle, G. L., \& Spain, W. J. (2004). Dynamic influences on coincidence detection in neocortical pyramidal neurons. L. Neurosci., 24(8), 1839-1851.

Gruber, T., Müller, M. M., Keil, A., \& Elbert, T. (1999). Selective visual-spatial attention alters induced gamma band responses in the human EEG. Clinical Neurophysioloou 110(12), 2074-2085.

Gutkin, B. S., \& Ermentrout, G. B. (1998). Dynamics of membrane excitability determine interspike interval variability: A link between spike generation mechanisms and cortical spike train statistics. Neural Comp., 10, 1047-1065.

Gutkin, B. S., Ermentrout, G. B., \& Reyes, A. D. (2005). Phase-response curves give the responses of neurons to transient inputs. L. Neurophysiol., 94, 1623-1635.

Hasselmo, M. E., \& McGaughy, J. (2003). High acetylcholine levels set circuit dynamics for attention and encoding and low acetylcholine levels set dynamics for consolidation. Progress in Brain Research, 145, 207-231.

Häusser, M., \& Clark, B. A. (1997). Tonic synaptic inhibition modulates neuronal output pattern and spatiotemporal synpatic integration. Neuron, 19, 665-678.

Hodgkin, A. L., \& Huxley, A. F. (1952). A quantitative description of membrane current and its application to conduction and excitation in nerve. J. Physiol. (London), $117,500-544$.

Hoppensteadt, F. C., \& Izhikevich, E. M. (1997). Weakly connected neural networks. New York: Springer-Verlag. 
Latham, P. E., Richmond, B. J., Nelson, P. G., \& Nirenberg, S. (2000). Intrinsic dynamics in neuronal networks. I. Theory. J. Neurophysiol., 83, 808-827.

Lumer, E. D. (2000). Effects of spike timing on winner-take-all competition in model cortical circuits. Neural Computation, 12, 181-194.

Lüthi, A., \& McCormick, D. A. (1998). H-current: Properties of a neuronal and network pacemaker. Neuron, 21, 9-12.

Mishra, J., Fellous, J.-M., \& Sejnowski, T. J. (2006). Selective attention through phase relationship of excitatory and inhibitory input synchrony in a model cortical neuron. Neural Networks, 19, 1329-1346.

Murthy, V. N., \& Fetz, F. E. (1994). Effects of input synchrony on the response of a three-conductance cortical neuron model. Neural Computation, 6, 1111-1126.

Olufsen, M., Whittington, M. A., Camperi, M., \& Kopell, N. (2003). New functions for the gamma rhythm: Population tuning and preprocessing for the beta rhythm. I. Comp. Neurosci., 14, 33-54.

Pouille, F., \& Scanziani, M. (2001). Enforcement of temporal fidelity in pyramidal cells by somatic feed-forward inhibition. Science, 293, 1159-1163.

Sarter, M., Givens, B., \& Bruno, J. P. (2001). The cognitive neuroscience of sustained attention: Where top-down meets bottom-up. Brain Res. Rev., 35, 146-160.

Singer, W. (1999). Neuronal synchrony: A versatile code for the definition of relations. Neuron, 24, 49-65.

Singer, W., \& Gray, C. M. (1995). Visual feature integration and the temporal correlation hypothesis. Ann. Rev. Neurosci., 18, 555-586.

Tateno, T., Harsch, A., \& Robinson, H. P. C. (2004). Threshold firing frequency-current relationships of neurons in rat somatosensor cortex: Type 1 and type 2 dynamics. L. Neurophysiol., 92, 2283-2294.

Taylor, K., Mandon, S., Freiwald, W. A., \& Kreiter, A. K. (2005). Coherent oscillatory activity in monkey area V4 predicts successful allocation of attention. Cerebral Cortex, 15(9), 1424-1437.

Tiesinga, P. H. E. (2002). From neuron to brain: Statistical physics of the nervous system. In A. Macias, F. J. Uribe, \& E. Diaz (Eds.), Developments in mathematical and experimental physics. Norwell, MA: Kluwer.

Tiesinga, P. H. E. (2005). Stimulus competition by inhibitory interference. Neural Computation, 17, 2421-2453.

Tiesinga, P. H. E., Fellous, J.-M., Salinas, E., José, J. V., \& Sejnowski, T. J. (2004). Synchronization as a mechanism for attentional gain modulation. Neurocomputing, 58-60, 641-646.

von der Malsburg, C., \& Schneider, W. (1986). A neural cocktail-party processor. Biol. Cubern., 54(1), 29-40.

White, J. A., Banks, M. I., Pearce, R. A., \& Kopell, N. J. (2000). Networks of inteneurons with fast and slow $\gamma$-aminobutyric acid type $A\left(\mathrm{GABA}_{\mathrm{A}}\right)$ kinetics provide substrate for mixed gamma-theta rhythm. Proc. Natl. Acad. Sci. USA $97(14), 8128-$ 8133.

Received July 25, 2006; accepted February 13, 2007. 
This article has been cited by: 